\title{
SEMIGROUPS WITH A DENSE SUBGROUP
}

\author{
BY
}

W. S. OWEN $\left({ }^{1}\right)$

\begin{abstract}
The purpose of this paper is twofold. First, it is shown that the ideal structure of a semigroup with dense subgroup is closely related to its transformation group structure. That is, if a left orbit through a given point is locally compact, then the members of this orbit are precisely those elements which generate the same left ideal as the given point.

Secondly, the author gives a number of theorems which have as their goal the establishment of a natural product structure near a nonzero idempotent. Specifically the work of F. Knowles [11] is improved upon to include (1) the possibility of a nonconnected group; (2) the possibility of a nonsimply connected orbit; and (3) the case in which the boundary of the group is more than a single orbit.
\end{abstract}

Introduction. Numerous papers ostensibly dealing with semigroups with identity on a manifold (and having their origins in [8]) have in reality dealt almost exclusively with the maximal group $G$ and its closure. In fact, for many of the results so obtained, one may as well have assumed merely that one had a dense Lie subgroup. No exhaustive study has been made of this hypothesis, nor does this paper in any way attempt to summarize what has been done in this area. Results have been obtained, for example, concerning the question of when an arbitrary dense subgroup is open, and these results are totally ignored in the present paper.

The emphasis in the present paper is twofold. First, it is shown that the transformation group structure inherent in the semigroup is intimately connected with its structure via Green's relations.

Second, several results are obtained which make it possible to give a topological decomposition of the semigroup analogous to that obtained in [10]. Specifically, the following is a special case of Theorem 14 of the paper: Let $S$ be a semigroup on a simply connected manifold. Suppose $S$ has a completely simple kernel $M$ and a dense connected Lie subgroup $G$. Let $e$ be an idempotent in $M$

Received by the editors April 2, 1973 and, in revised form, December 19, 1973 and July 22, 1974.

$A M S(M O S)$ subject classifications (1970). Primary 20M10, 22A15; Secondary 54H15, 57E99.

Key words and phrases. Completely semisimple semigroup, dense Lie subgroup, Green's relations, local cross section, locally compact orbit.

(1) This paper is derived from a portion of the author's doctoral dissertation which was written at the University of Georgia under the direction of J. G. Horne. 
with $G e, e G$, and $e G_{L}(e)$ locally compact. Then $S$ is homeomorphic to

$$
[S e \cap E(S)] \times e S e \times P^{-} \times[e S \cap E(S)]
$$

where $P=G_{L}(e) \cap G_{R}(e)$. It should be remarked that Theorem 14 also gives a significant generalization of Theorem 5 of [7]. For in the latter, Knowles considers only semigroups in which the boundary of $G$ is a single orbit $\mathrm{Ge}$. It is easily seen that the boundary in this case is a left group (see [1]) and hence is the completely simple kernel of $S$. Had Theorem 14 been available prior to work on [5], it could have made the work therein much easier.

Even if $S$ has a zero, in which case Theorem 14 is of no value, one has at least a local version of the above product structure near nonzero idempotents. In fact, Theorem 11 handles as well the case in which $S$ need not be simply connected nor have a completely simple kernel.

Notation. $G$ will always be a dense topological subgroup of the semigroup $S$, and $G_{0}$ denotes the identity component of $G . L=\partial G$ denotes $S-G$, and $e$ is an idempotent in $L . H=G_{L}(e)$ denotes the left isotropy group of $e$, and $K=$ $G_{R}(e)$ denotes the right isotropy group of $e . P$ will denote $H \cap K$.

We shall use $J, L$, and $R$ to denote Green's relations: namely, $a J b$ if and and only if $a$ and $b$ generate the same two-sided ideal. $L$ and $R$ are the one-sided analogues. $J_{a}$ will denote the $J$-class containing $a$. The meanings of $L_{a}$ and $R_{a}$ are analogous. $H(e)$ denotes the maximal subgroup containing $e . E(S)$ denotes the set of idempotents of $S$.

We let $A=\{g \mid g e \in H(e)\}, B=\{g \mid e g \in H(e)\}$, and $C=A \cap B$. Observe that $H \cap B=P$ and that $K \cap A=P$.

We begin with a theorem which links the ideal structure of $S$ with the transformation group structure. We make use of the following well-known result from topology (see [2, p. 255, Exercise 7]): If $X$ is a locally compact, dense subset of a space $Y$, then $X$ is open in $Y$.

REMARK. $G x$ is dense in $S x$. Thus if $G x$ is locally compact, it is open in Sx.

THEOREM 1. Let $G$ be a dense subgroup of $S$, and suppose $x \in S$ is such that $G x$ is locally compact. Then the orbit $G x$ coincides with the L-class containing $x$. In particular, if $x \neq 0$ and if $S x$ is a 0 -minimal left ideal, then $G x=$ $S x-\{0\}$.

Proof. If $y \in G x$, then $y \in S x$ so $S y \subseteq S x$. But also $x \in G y$ so $S x \subseteq S y$. Thus if $y \in G x$, then $S y=S x$, so that $y \in L_{x}$. Conversely, suppose $y \in L_{x}$, so that $S y=S x$. Now $G y$ is dense in $S y=S x$. If $y \notin G x$, then $G y \cap G x=\varnothing$, i.e., 
Gy $\subset S x-G x$. Since $G y$ is dense in $S x$, this would give $S x-G x$ dense in $S x$. But since $G x$ is locally compact and dense in $S x, G x$ is open in $S x$, so $S x-G x$ is closed in $S x$. Thus $S x-G x=S x$. But since $G x \neq \varnothing$, this is impossible. Hence if $y \in L_{x}$, then $y \in G x$, so that $L_{x}=G x$.

Now suppose $S x$ is a 0 -minimal left ideal of $S$. If $y \in S x-\{0\}$, either $S y=\{0\}$ or $S y=S x$. But since $S$ has an identity, $S y \neq 0$, so $y \in L_{x}=G x$. Thus in this case $G x=S x-\{0\}$.

A frequent hypothesis below is that $H(e)=J_{e} \cap e S e$. We remark that this is true if $S e$ is left minimal or left 0 -minimal. Note also that if $S$ is completely semisimple (see [1, vol. II, p. 32] for definition and notation), this property holds for every idempotent $e$. For certainly $H(e) \subset J_{e} \cap e S e$. Conversely, let $\nu$ : $J(e) \rightarrow J(e) / I(e)$ be the natural map, and let $G^{*}=\nu(e S e)$. Then, since $J(e) / I(e)$ is completely 0-simple, $G^{*}$ is a group with zero. Since $J_{e}=J(e)-I(e), v\left(J_{e}\right) \cap$ $G^{*}$ is a group, whence $J_{e} \cap e S e$ is a group. Since $H(e)$ is the maximal group, $H(e)=J_{e} \cap e S e$.

Corollary 2. Let $G$ be a dense subgroup of $S$, and let $e \in E(S)$ be such that $H(e)=J_{e} \cap e S e$ and such that Ge is locally compact. Then

(1) $H(e)$ is open in eSe,

(2) $H(e)$ is closed in $L_{e}=G e$, and

(3) $H(e)$ is a topological group.

Proof. Since $H(e) \subset L_{e} \cap e S e \subset J_{e} \cap e S e$ (and similarly for $R_{e}$ ), if $H(e)=$ $J_{e} \cap e S e$, then $H(e)=L_{e} \cap e S e=R_{e} \cap e S e$. (1) follows from the fact that $L_{e}=G e$ is open in $S e$, and the fact that $e S e=S e \cap e S$. (2) follows from the fact that $e S e$ is closed in $S$. To get (3), recall that $H(e)$ is closed in $G e$ and hence locally compact. By [3], any such group is a topological group.

Proposition 3. Let $G$ be a dense subgroup of $S$, let $e$ be any idempotent such that $G e$ and $e G$ are locally compact, and let $A=\{g \in G: g e \in H(e)\}$. Then

(1) $A$ is a subgroup of $G$,

(2) the map $g \rightarrow g e$, with $g \in A$, is a homomorphism of $A$ onto $H(e)$ with kernel $G_{L}(e)$, and

(3) if $H(e)=J_{e} \cap e S e$, then $A$ is closed in $G$.

Proof. In [5], Horne defines $G^{L}(e)=\{g \in G: g e \in e G\}$, and observes that $G^{L}(e)$ is a subgroup, and that $G^{L}(e) \cdot e=G e \cap e G$. He also observes that the latter is a group. We wish to see that $H(e)=G e \cap e G$, so that $G^{L}(e)=A$. Since $G e \cap e G$ is a subgroup of $e S e$, certainly $G e \cap e G \subset H(e)$ since $H(e)$ is the (unique) maximal subgroup of $e S e$. On the other hand, it is well known (see, for 
example, [1]) that $H(e) \subset L_{e} \cap R_{e}$, which equals $G e \cap e G$ by Theorem 1. Thus $H(e)=G e \cap e G$. This establishes (1). The proof of (2) is straightforward and is omitted. For (3), recall that if $H(e)=J_{e} \cap e S e$, then $H(e)$ is closed in $G e$ (Corollary 2). If $f(s)=s e$, then $A=f^{-1}(H(e))$, which is therefore closed.

We now seek to generalize Theorems 1 and 2 of [6], and also to give some natural conditions which put us in a situation to apply this generalized version of the latter.

THEOREM 4. Let $G$ be a locally compact, o-compact group of finite dimension which is dense in a semigroup $S$. If $e$ is an idempotent such that Ge is locally compact, then $e \in G_{L}(e)^{-}$.

ProOF. We need only inspect Knowles' proof to discover that the only time connectivity of $G$ is used is to get $G e$ homeomorphic to $G / G_{L}(e)$. From Theorem I.2.5 of [4], this will be true provided $G$ is locally compact, $\sigma$-compact and provided $G e$ is locally compact.

THEOREM 5. Let $G$ be a dense subgroup of a semigroup $S$, and let $e$ be an idempotent. Suppose $V$ is a subspace of $G$ such that the map $v \rightarrow v e$ is a homeomorphism of $V$ onto Ge. Then the map $m: V \times G_{L}(e) \rightarrow G$ is a homeomorphism. Moreover, if $\partial G=G e$, then

(i) $G_{L}(e)^{-}=G_{L}(e) \cup\{e\}$, and

(ii) $m: V \times G_{L}(e)^{-} \rightarrow G^{-}$is a homeomorphism.

Proof. Since $V e=G e$, certainly $G=V \cdot G_{L}(e)$. Suppose $v_{1} h_{1}=v_{2} h_{2}$ with $v_{i} \in V, h_{i} \in G_{L}(e)$. Then $v_{1} e=v_{1} h_{1} e=v_{2} h_{2} e=v_{2} e$, so $v_{1}=v_{2}$. Then $h_{1}=h_{2}$, so $m: V \times G_{L}(e) \rightarrow G$ is $1-1$ and onto. If $v_{i} h_{i} \rightarrow v h$, then $v_{i} e \rightarrow$ $v e$, so $v_{i} \rightarrow v$, whence $h_{i} \rightarrow h$. This establishes the first statement. The remainder of the theorem is a generalization of Theorem 2 of [6]. The only nonobvious ingredient of Knowles' proof is the fact that $e \in G_{L}(e)^{-}$. To see this, let $v_{i} h_{i} \rightarrow$ $e$; then $v_{i} e \rightarrow e$, so $v_{i} \rightarrow 1$, and $h_{i} \rightarrow e$.

LEMMA 6. Let $G$ be a dense subgroup of $S$, and let $M$ be a completely 0 simple ideal in $\partial G$. Suppose there is an element $x \neq 0$ in $M$ such that $G x$ and $x G$ are locally compact. Then if $y \in M-\{0\}, G y$ and $y G$ are homeomorphic to $G x$ and $x G$, respectively, and in particular are locally compact.

Proof. By Theorem 6.25 of [1], $S x=M x$ is a 0 -minimal left ideal of $S$. Thus by Theorem 1, $S x=G x \cup\{0\}$. Since $S x \cap y S \neq 0$ [1], $G x \cap y S \neq \varnothing$. Let $z \in G x \cap y S$. Then $z=g x$ for some $g \in G$, so $z G=(g x) G=g(x G)$ which is homeomorphic to $x G$. Thus $z G$ is locally compact, so $z G=z S-\{0\}$ by the 
dual to Theorem 1. Since $z \in y S, z S=y S$, so $y \in z S-\{0\}=z G$, so $y G=z G$ is locally compact, and homeomorphic to $x G$. Dually, $G y$ is homeomorphic to Gx.

The following is of interest in its own right, as well as being an ingredient of what is to come.

Proposition 7. Let $G$ be a locally compact, o-compact group of finite dimension, which is dense in a semigroup $S$. Let $M$ be a completely 0 -simple ideal in $\partial G$, and let $e$ be a nonzero idempotent in $M$ such that $G e$ and $e G$ are locally compact. Then $M \cap \partial H=e G \cap E(S)$, where $H=G_{L}(e)$. In particular, if $M=$ $\partial G$, then $\partial H=e G \cap E(S)$.

Proof. Let $f \in e G \cap E(S)$. Since $f \in e G, G_{L}(f)=G_{L}(e)$. Moreover, $G f$ is locally compact by the above lemma, so $f \in G_{L}(f)^{-}=G_{L}(e)^{-}$by Theorem 4 . Certainly $f \in M$; hence $e G \cap E(S) \subseteq M \cap \partial\left(G_{L}(e)\right)$.

Conversely, let $x \in M \cap \partial H$. Since $e$ is a right zero for $H^{-}$, xe $=e$, so $e \in x S$, which implies that $e S \subseteq x S$. Since $x \in M, x S$ is a 0 -minimal right ideal, so $e S=x S$, so $x \in e S$. Since $e$ is a left identity for $e S$, ex $=x$. But then $x^{2}=$ $(e x)(e x)=e(x e) x=e^{2} x=e x=x$, so $x$ is idempotent. Hence $x \in e S \cap E(S)$. Since $e G=e S-\{0\}$ (Theorem 1) and since $x \neq 0, x \in e G \cap E(S)$. Thus $M \cap$ $\partial H=e G \cap E(S)$.

Terminology. We say $W$ is a local cross section (1.c.s.) to $H$ in $G$, provided the map $m: W \times H \rightarrow G$ is a homeomorphism onto a neighborhood of 1 in $G$.

LEMMA 8. Let $S$ be a semigroup satisfying the hypotheses of Theorem 4. Then $H^{-}=\{s \mid s e=e\}$. Moreover, if $e H$ is locally compact, then $P^{-}=\{s \mid s e=$ es $=e\}$, where $P=G_{L}(e) \cap G_{R}(e)$.

Proof. Let $W$ be a local cross section to $H$ in $G$. Then the map $w \rightarrow$ we is a homeomorphism of $W$ onto a neighborhood of $e$ in $G e$. Clearly $\mathrm{H}^{-} \mathrm{C}$ $\{s \mid s e=e\}$. Now suppose $s e=e$. Let $g_{i} \rightarrow s, g_{i} \in G$. Then $g_{i} e \rightarrow s e=e$, so eventually $g_{i} e=w_{i} e$ for some $w_{i} \in W$. Let $h_{i}=w_{i}^{-1} g_{i}$. Then $h_{i} \in H$ and $g_{i}=$ $w_{i} h_{i}$. Since $w_{i} e=g_{i} e \rightarrow e, w_{i} \rightarrow 1$ so $h_{i} \rightarrow s$. Thus $s \in H^{-}$, as desired.

The second statement is an application of the first to the semigroup $G_{L}(e)^{-}$, with $H$ acting on $\partial H$ on the right. The isotropy for this action is $G_{L}(e) \cap$ $G_{R}(e)=P$, so by what we have just shown, $P^{-}=\left\{s \in G_{L}(e)^{-}: e s=e\right\}$. Clearly the latter set is $\{s: s e=e s=e\}$.

Lemma 9. Suppose $S$ is a semigroup, and $e \in E(S)$ is such that $H(e)$ is an open topological subgroup of eSe. Then the multiplication map $m:\left[L_{e} \cap E(S)\right] \times$ $H(e) \rightarrow S e$ is an isomorphism onto a neighborhood of $e$ in Se. 
Proof. Observe that $L_{e} \cap E(S)$ is a left trivial semigroup. Let $a_{i}, a \in$ $L_{e} \cap E(S)$, and let $x_{i}, x \in H(e)$. Suppose $a_{i} x_{i} \rightarrow a x$. Multiplication by $e$ on the left gives $x_{i} \rightarrow x$. Since $H(e)$ is a topological group, $x_{i}^{-1} \rightarrow x^{-1}$, so $a_{i}=a_{i} e \rightarrow$ $a e=a$. Similarly, if $a_{1} x_{1}=a_{2} x_{2}, x_{1}=x_{2}$ so $a_{1}=a_{2}$. Thus the map $m:\left[L_{e} \cap\right.$ $E(S)] \times H(e) \rightarrow S e$ is a homeomorphism.

The proof that the map is algebraically an isomorphism is trivial and is omitted.

To see that the image is a neighborhood of $e$ in $S e$, let $s_{i} e \rightarrow e$. Then $e s_{i} e \rightarrow e$, so eventually $e s_{i} e \in H(e)$, since $H(e)$ is open in $e S e$. Moreover, we wish to see that $s_{i} e\left(e s_{i} e\right)^{-1} \in L_{e} \cap E(S)$. Certainly $s_{i} e\left(e s_{i} e\right)^{-1} \in E(S)$. To see that $s_{i} e\left(e s_{i} e\right)^{-1}$ generates $S e$, it suffices to show that $e \in S\left[s_{i} e\left(e s_{i} e\right)^{-1}\right]$. Since $e=e\left[s_{i} e\left(e s_{i} e\right)^{-1}\right]$, this is clear. Thus $s_{i} e\left(e s_{i} e\right)^{-1} \in L_{e} \cap E(S)$. Since $s_{i} e=$ $s_{i} e\left(e s_{i} e\right)^{-1} e s_{i} e$, we are done.

LEMma 10. Suppose $S$ is a semigroup having a dense subgroup which is locally compact, o-compact, and finite dimensional. Suppose $e \in \partial G$ is such that $H(e)=J_{e} \cap e S e$, and such that $G e$ and $e G$ are locally compact. Then the following are equivalent:

(1) $\mathrm{eH}$ is locally compact,

(2) $\mathrm{Ke}$ is locally compact,

(3) $B H$ is open in $G$,

(4) $K A$ is open in $G$.

Moreover, if these conditions are satisfied, then

(5) $e H$ is open in $e S \cap E(S)$,

(6) $\mathrm{Ke}$ is open in $\mathrm{Se} \cap \mathrm{E}(\mathrm{S})$,

(7) $\mathrm{Ce}$ is open in $\mathrm{Ae}=H(e)$.

Proof. (1) $\Rightarrow$ (3) and (5). First observe that $e G \cap E(S)=R_{e} \cap E(S)$ is right trivial and open in $e S \cap E(S)$. Since every member of $e G \cap E(S)$ is in $H^{-}$ (Lemma 8), it is easily seen that $e H$ is dense in $e G \cap E(S)$. It follows that $e H$ is open in $e G \cap E(S)$, and hence in $e S \cap E(S)$. Now $e B=H(e)$, so by the previous lemma it follows that $e B e H=e B H$ is open in $e S$ and hence in $e G$. Thus $B H$ is open in $G$.

(3) $\Rightarrow$ (4) and (7). Recall that we set $C=A \cap B$. Since $B H$ is open in $G$, $A \cap B H=C H$ is open in $A$. Since the map $g \rightarrow g e$ is open [3], this means $C H e=C e$ is open in $A e=H(e)$. Now observe that $K A$ is open in $G$ if and only if $K A e$ is open in $G e$. Thus we need to show that if $g_{i} e \rightarrow e$, eventually $g_{i} e \in$ $K A e$. Since $B H e=B e$ is open in $G e$, we may as well assume $g_{i} \in B$. By the lemma, eventually $g_{i} e \in[G e \cap E(S)] \cdot C e$. We abbreviate $g_{i} e$ by $g e$ and write $g e=$ 
sce where $s \in G e \cap E(S)$ and $c \in C$. Let $k=g c^{-1}$. We wish to see that $k \in K$. But $e k=e g c^{-1}=e g e c^{-1} e=e s c e c^{-1} e=e s e=e$ since both $g$ and $c$ are in $B$. Thus $k \in K$, as desired. Then $g e=k c e \in K C e \subset K A e$, so $K A e$ is open in $G e$, whence $K A$ is open in $G$.

(4) $\Rightarrow$ (2) and (6). As we have seen, $K A$ is open in $G$ if and only if $K A e=$ $K e A e$ is open in $G e$. Since $K e \subset G e \cap E(S)$ and $A e=H(e)$, it follows from the previous lemma that $K A e$ is homeomorphic to $(K e) \times(A e)$. Thus if $K A e$ is locally compact, so is $K e$.

(4) $\Rightarrow(6)$ is identical to (1) $\Rightarrow(5)$.

The remaining equivalences follow by dualizing the above.

The following theorem gives us the local product structure referred to in the introduction.

THEOREM 11. Let $S$ be a semigroup satisfying the hypotheses of the lemma, and any of conditions (1)-(4) therein. Then there is a neighborhood of $e$ in $S$ locally homeomorphic to $[G e \cap E(S)] \times H(e) \times P^{-} \times[e G \cap E(S)]$, or alternately, to $K / P \times A / H \times P^{-} \times H / P$.

Proof. We showed in the lemma that $K e$ is open in $G e \cap E(S)$ and that $e H$ is open in $e G \cap E(S)$. It follows that $K / P$ and $H / P$ are locally homeomorphic to $G e \cap E(S)$ and $e G \cap E(S)$, respectively. Certainly $A / H$ is isomorphic to $H(e)$ by Proposition 3.

Now let $R$ be a local cross section (1.c.s.) of $P$ in $K$, let $T$ be a 1.c.s. of $P=$ $H \cap B$ in $C=A \cap B$, and let $Q$ be a l.c.s. of $P$ in $H$. Then the maps $r \rightarrow r e$ $(r \in R)$ and $q \rightarrow e q(q \in Q)$ are homeomorphisms onto open subsets of $G e \cap$ $E(S)$ and $e G \cap E(S)$, respectively. Moreover, since $C e$ is open in $A e=H(e)$ (Lemma 10$), C / P$ is isomorphic to $C e$ (an open subgroup of $H(e)$ ), to the map $t \rightarrow$ te $(t \in T)$ is likewise a homeomorphism of $T$ onto an open subset of $H(e)$. We shall show that the multiplication map $m: R \times T \times P^{-} \times Q \rightarrow S$ is a homeomorphism onto a neighborhood of $e$ in $S$.

(1) To see that $m$ is a homeomorphism, it suffices to see that if $r_{i} t_{i} p_{i} q_{i} \rightarrow$ $r t p q$ (with $r_{i}, r \in R ; t_{i}, t \in T ; p_{i}, p \in P^{-}$; and $q_{i}, q \in Q$ ), then $r_{i} \rightarrow r, t_{i} \rightarrow t$, $p_{i} \rightarrow p$, and $q_{i} \rightarrow q$. (The case in which all these nets are constant yields the one-to-oneness of $m$.) Recall that $R \subset G_{R}(e), Q \subset G_{L}(e), P=G_{L}(e) \cap G_{R}(e)$, and $T \subset A \cap B$. Thus $r_{i} t_{i} e=r_{i} t_{i} p_{i} q_{i} e \rightarrow r t p q e=r t e$, so that $e t_{i} e=e r_{i} t_{i} e \rightarrow$ erte $=$ $e t e=t e$. But then, since $t_{i} e, t e \in H(e),\left(e t_{i} e\right)^{-1}=\left(t_{i} e\right)^{-1} \rightarrow(t e)^{-1}$ so $r_{i} e \rightarrow$ $r e$, whence $r_{i} \rightarrow r$. Likewise $t_{i} \rightarrow t$ so $p_{i} q_{i} \rightarrow p q$. Therefore $e q_{i} \rightarrow e q$ so $q_{i} \rightarrow q$ so $p_{i} \rightarrow p$, as desired.

(2) We next show that $R \cdot T \cdot P^{-} \cdot Q$ is a neighborhood of $e$ in $S$ by showing that if $s_{i} \rightarrow e$, eventually $s_{i} \in R \cdot T \cdot P^{-} \cdot Q$. So suppose $s_{i} \rightarrow e$. Then 
eventually $e s_{i} e \in T e$, say $e s_{i} e=t_{i} e$. Moreover, $s_{i}\left(e s_{i} e\right)^{-1} \in S e \cap E(S)$ and $\left(e s_{i} e\right)^{-1} s_{i} \in e S \cap E(S)$, so likewise, eventually $s_{i}\left(e s_{i} e\right)^{-1} \in R e$ and $\left(e s_{i} e\right)^{-1} s_{i} \in$ $e Q$. Let us set $s_{i}\left(e s_{i} e\right)^{-1}=r_{i} e$ and $\left(e s_{i} e\right)^{-1} s_{i}=e q_{i}$. Henceforth we assume $i$ is large enough so that all the above containments hold. We shall drop the subscript $i$ for convenience. Then se $=r e(e s e)=$ rete $=r t e$ so $t^{-1} r^{-1} s \in\{s \mid s e=e\}=$ $G_{L}(e)^{-}$by Lemma 8. Let $p=t^{-1} r^{-1} s$. Then $e p=e t^{-1} r^{-1} s=e t^{-1} e r^{-1} s=$ $(e s e)^{-1} e s=(e s e)^{-1} s=e q$, so likewise $p q^{-1} \in G_{R}(e)^{-}$. Let $p_{1}=p q^{-1}$. Since both $p$ and $q$ belong to $G_{L}(e)^{-}$, so does $p_{1}$. Thus $p_{1} \in P^{-}$by Lemma 8. Then $p=p_{1} q=t^{-1} r^{-1} s$ so $s=r t p_{1} q \in R \cdot T \cdot P^{-} \cdot Q$, as desired.

LEMMA 12. Let $S$ be a semigroup satisfying the hypotheses of the previous theorem. Suppose further that $S$ is locally arc-wise connected. Then there is an arc in $P^{-}$running from 1 to $e$.

Proof. By the theorem, $P^{-}$is locally arc-wise connected at $e$. Thus there is an $\operatorname{arc} I$ in $P^{-}$running from $e$ to some point $x$ of $P$. Since $e$ is a zero for $P^{-}$, $x^{-1} I$ is an arc in $P^{-}$running from $e$ to 1 .

Notation. With $S$ as above, the arc guaranteed by the lemma will be denoted $[1, e]$.

LEMMA 13. Let $G$ be a locally connected group and $H$ a connected, normal subgroup such that $G / H$ is connected. Then $G$ is connected.

Proof. Let $\nu: G \rightarrow G / H$ be the natural map. Then $\nu\left(G_{0}\right)$ is an open (hence closed) subgroup of $G / H$, so $\nu\left(G_{0}\right)=G / H$. Then $G=G_{0} \cdot H$. But since $H$ is connected, $H \subset G_{0}$, so $G=G_{0}$.

Notation. Let $G_{0}$ denote the identity component of $G . H_{1}$ will denote $H \cap G_{0}, K_{1}=K \cap G_{0}$, and $P_{1}=P \cap G_{0}$. Likewise let $A_{1}=A \cap G_{0}, B_{1}=$ $B \cap G_{0}$, and $C_{1}=C \cap G_{0}$.

THEOREM 14. Let $S$ be a locally arc-wise connected, simply connected semigroup with a completely simple kernel $M$. Suppose further that $S$ has a dense Lie subgroup $G$ which is $\sigma$-compact (i.e. has a countable number of components). Let $e$ be an idempotent in $M \cap \partial G_{0}$ such that $G e, e G$, and $e H$ are locally compact. Then $S$ is homeomorphic to $[S e \cap E(S)] \times e S e \times P^{-} \times[e S \cap E(S)]$.

Proof. Since $M$ is completely simple, $M=S e S$, and $S e$ and $e S$ are minimal left and right ideals, respectively [1]. Thus by Theorem $1, G e=S e$ and $e G=e S$. Moreover, $S e$ and $e S$ are retracts of $S$ and hence are connected and simply connected. We wish to see that $G_{0} e=G e$ (and likewise $e G_{0}=e G$ ). Observe that $G_{0} e$ is open in $G e$ since $G_{0}$ is open in $G$. In fact, for each $x \in G, x G_{0} e$ is open in $G e$. Also, if $y \in G$ and $y G_{0} e \cap x G_{0} e \neq \varnothing$, then $G_{0} y e \cap G_{0} x e=\varnothing$ (since 
$y G_{0}=G_{0} y$ and $x G_{0}=G_{0} x$ ), whence $y G_{0} e=G_{0} y e=G_{0} x e=x G_{0} e$. Thus the sets $x G_{0} e \quad(x \in G)$ are equal or disjoint. Since $G e$ is connected, it follows that $G_{0} e=G e$. Thus $G_{0}$ is a connected group acting with simply connected orbit. By [9], this means $H_{1}$ is connected. Likewise, $K_{1}$ is connected.

Since $J_{e}=M$ and $H(e)=e S e \subset M$, certainly $H(e)=J_{e} \cap e S e$. Thus by Corollary 2, eSe is a topological group. Now by [10], $M$ is homeomorphic to $[\mathrm{Se} \cap E(S)] \times e S e \times[e S \cap E(S)]$. Since $M$ is connected, so are each of $S e \cap$ $E(S)$, eSe, and $e S \cap E(S)$. Also, $e S e$ is simply connected, as a retract of $S e$.

By Lemma $10, e H$ is open in $e S \cap E(S)$. Likewise, $K e$ is open in $S e \cap$ $E(S)$. Moreover, every member of $e S \cap E(S)$ is in $e G$ and so has $H$ as its isotropy group. Thus every orbit $f H(f \in e S \cap E(S))$ is open in $e S \cap E(S)$. Since $e S \cap$ $E(S)$ is connected, $e H=e S \cap E(S)$. Likewise, $K e=S e \cap E(S)$. Also by Lemma $10, C e$ is an open (hence closed) subgroup of $H(e)=e S e$, so we have $C e=e S e$.

Now $C e=e S e$ is a factor of $G e=[S e \cap E(S)] \times e S e$, and hence is locally compact. Thus the map $c \rightarrow c e$ is open. Since $C_{1}=C \cap G_{0}$ is open in $C$, $C_{1} e$ is an open (hence closed) subgroup of $e S e$, so as above $C_{1} e=e S e$.

We wish to see that $K e$ and $e H$ are contractible. Define $F:[1, e] \times K e \rightarrow$ $K e$ by $F(p, k e)=p k e$. (Observe that $p k e \in S e \cap E(S)=K e$.) Then $F$ is a homotopy from the identity map on $K e$ to the constant $\{e\}$, so $K e$ is a contractible manifold. Dually, $e H$ is contractible. Thus by [11] there is a global cross section $R$ of $P_{1}$ in $K_{1}$, and we write $K_{1}=R P_{1}$. Dually $H_{1}=P_{1} Q$ for some cross section $Q$ of $P_{1}$ in $H_{1}$.

To get a global cross section of $P_{1}=H_{1} \cap B_{1}$ in $C_{1}$, we must show that the latter is connected. To see this, recall that $e C_{1}=H(e)$, so that $K_{1} C_{1}=B_{1}$. Also observe that $B_{1}$ is a Lie subgroup since $B_{1}$ is open in $B$ which is closed in $G$. But then $B_{1} e=K_{1} C_{1} e=K_{1} e C_{1} e=[S e \cap E(S)] \cdot e S e=G e$ is simply connected. Since $e S e=B_{1} / K_{1}$ is connected and since $K_{1}$ is connected, this means $B_{1}$ is connected by Lemma 13. But then $B_{1} \cap H_{1}=P_{1}$ is connected (since $B_{1} e$ is simply connected). Since $e S e=C_{1} / P_{1}$ is connected, applying Lemma 13 again we see that $C_{1}$ is connected. Thus by [7], there is a global cross section $T$ of $P_{1}$ in $C_{1}$.

Then the maps $r \rightarrow r e, t \rightarrow t e$, and $q \rightarrow e q$ are homeomorphisms of $R$ onto $S e \cap E(S)$, of $T$ onto $e S e$, and of $Q$ onto $e S \cap E(S)$, respectively. By Theorem 5 , it follows that $R$ is a cross section of $P$ in $K$, that $T$ is a cross section of $P$ in $C$, and that $Q$ is a cross section of $P$ in $H$. Thus the local homeomorphism constructed in the proof of Theorem 11 is actually a global homeomorphism in the present case. We wish to see that the image is all of $S$ (not merely a neighborhood of $e$ in $S$ ). Let $s \in S$. Now $R e=S e \cap E(S), T e=e S e$, and $e Q=$ $e S \cap E(S)$. Let ese $=t e, s(e s e)^{-1}=r e$, and $(e s e)^{-1} s=e q$, where $t \in T, r \in R$, 
and $q \in Q$. Exactly as in Theorem 11 (proof, part (2)), $s=r t p_{1} q \in R \cdot T \cdot$ $P^{-} \cdot Q$, where $p_{1}=t^{-1} r^{-1} s q^{-1}$. Thus $S=R \cdot T \cdot P^{-} \cdot Q$, which is homeomorphic to [Se $\cap E(S)] \times e S e \times P^{-} \times[e S \cap E(S)]$. This concludes the proof.

It should be remarked at this point that if $S$ has a 0 , then necessarily $M=$ $\{e\}=\{0\}$ in the statement of the theorem. In this case, of course, $P=G$ and the theorem is trivial. Nevertheless, this theorem appears to have wide application of a nontrivial nature. In particular, one possible application is for semigroups on a half- $n$-space of arbitrary dimension (see [5] for definition). The author will show the relevance of this theorem in a sequel to the present paper.

\section{REFERENCES}

1. A. H. Clifford and G. B. Preston, The algebraic theory of semigroups. Vols. I, II, Math. Surveys, no. 7, Amer. Math. Soc., Providence, R. I., 1961, 1967. MR 24 \#A2627; 36 \#1558.

2. J. Dugundji, Topology, Allyn and Bacon, Boston, Mass., 1966. MR 33 \#1824.

3. R. Ellis, $A$ note on the continuity of the inverse, Proc. Amer. Math. Soc. 8 (1957), 372-373. MR 18, 745 .

4. G. Hochschild, The structure of Lie groups, Holden-Day, San Francisco, Calif., 1965. MR 34 \#7696.

5. J. G. Horne, Jr., Semigroups on a half-space, Trans. Amer. Math. Soc. 147 (1970), 1-53. MR 41 \#378.

6. F. Knowles, Semigroups that are the union of a group on $E^{3}$ and a plane, Trans. Amer. Math. Soc. 160 (1971), 305-325. MR 43 \#7545.

7. - Idempotents in the boundary of a Lie group, Pacific J. Math. 44 (1973), 191-200. MR 47 \#3592.

8. P. S. Mostert and A. L. Shields, Semigroups with identity on a manifold, Trans. Amer. Math. Soc. 91 (1959), 380-389. MR 21 \#4204.

9. G. D. Mostow, The extensibility of local Lie groups of transformations and groups on surfaces, Ann. of Math. (2) 52 (1950), 606-636. MR 14, 18.

10. W. S. Owen, The Rees theorem for locally compact semigroups, Semigroup Forum 6 (1973), 133-152.

11. N. E. Steenrod, The topology of fibre bundles, Princeton Math. Ser., vol. 14, Princeton Univ. Press, Princeton, N. J., 1951. MR 12, 522.

DEPARTMENT OF MATHEMATICS, UNIVERSITY OF GEORGIA, ATHENS, GEORGIA 30602

DEPARTMENT OF MATHEMATICS, UNIVERSITY OF AUBURN, AUBURN, ALABAMA 36830

Current address: 3377 Homer Place, Decatur, Georgia 30032 